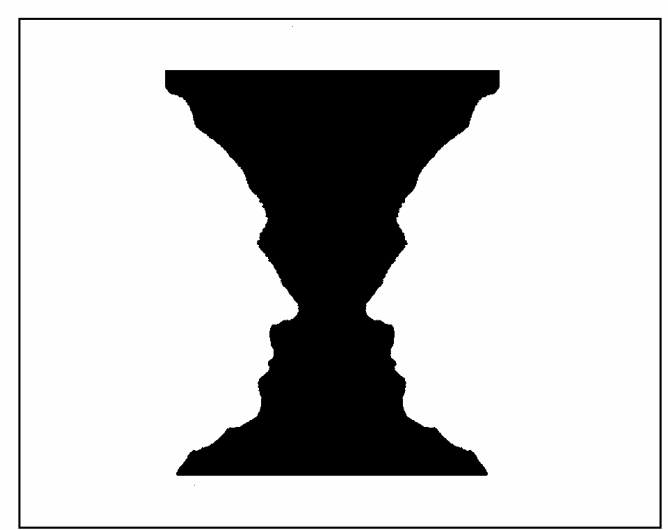

published by

www.semverteilung.vwl.uni-muenchen.de

Printed in Austria

\section{Local Aggregation in a Dynamic Setting}

By

Ekkehart Schlicht, Darmstadt, F. R. G.*

(Received July 14, 1989; revised version received February 2, 1990)

\section{Introduction}

Aggregation is concerned with the reduction of dimensions: a high-dimensional "micro-system" is to be described in lower dimensions by an appropriately chosen "macro-system". If we confine our attention to the neighborhood of equilibria, we may linearize the systems involved and obtain a local, or linear, aggregation problem.

The aggregation problem is obviously of great importance. ${ }^{1}$ Many of our theories such as business cycle theories or international trade theories are usually conceived as macro theories from the beginning; these are the only tools we have to deal with issues of business cycles and international trade, and the problem arises how these theories may be related to the "true" but unknown microeconomic processes the macro surface of which they are intended to describe. This is the "hermeneutic aggregation problem" which is pervasive in economic theory. ${ }^{2}$ On the other hand, many empirical statistics refer to aggregate observations. This renders the estimation of truly microeconomic models unfeasible. They comprise too many parameters as compared to the

* An earlier version of the paper (Schlicht, 1985 b) was presented at the Econometric Society European Meeting in Budapest (Hungary) 1986. Comments by anonymous referees have been extremely helpful. This paper is dedicated to my wife.

${ }^{1}$ See e. g. Fisher (1969, Chap. 1) and Schlicht (1977, Chap. 2; 1985 a, Chap. 5).

${ }_{2}^{2}$ Schlicht (1977, pp. 29 and 93; 1985 a, pp. 10 and 93). 
number of available observations, and misspecification errors multiply. We can only look at the aggregative consequences of these microeconomic models, and this involves aggregation. The problem will, I think, remain with us for a long time even if our computers become increasingly faster. To describe any state of the world in complete microeconomic detail is even more difficult technically than drawing a map on a $1: 1$ scale - and probably equally useless. ${ }^{3}$

The first approaches to the aggregation problem in the context of economic theory (going back to a famous exchange between Lawrence Klein and Kenneth May in the forties) tried to establish exact aggregation procedures but it became clear quite soon that this will not lead to practically useful results. As a consequence, the aggregation problem was posed in a different vein; it was conceived as a problem of optimal approximation: how to devise a macro system such that its behavior approximates the behavior of the underlying micro system as well as possible. This approach has been initiated by Fisher. ${ }^{4}$ The present paper pursues this line of argument and looks for approximate rather than exact aggregation.

Fisher was basically concerned with the problem of describing a high-dimensional linear system, say $y=A \cdot x$, in terms of a lowerdimensional macro system $Y=C \cdot X$, where the vectors $X$ and $Y$ are conceived as macro descriptions of the corresponding micro variables $x$ and $y$ and where the true micro system matrix $A$ leads to the system matrix $C$ in the aggregate model. This may be described as a static aggregation problem.

A quite different set of issues arise, however, if we look at the aggregation of dynamical systems, and this is the topic of the present paper. We shall consider a linear differential equations system

$$
\dot{x}=A \cdot(x-\bar{x}), x, \dot{x} \in \mathbb{R}^{n}:
$$

$A$ of order $n \times n$ and rank $n$;

and an aggregation rule

$$
X=B \cdot x, X \in \mathbb{R}^{m}, m<n ;
$$

$B$ of order $m \times n$ and rank $m$;

3 This analogy is, I think, due to Joan Robinson.

4 See Fisher (1962, 1969), Ijiri (1971) and Schneeweiß (1965). The excellent paper by Sondermann (1973) contains a lucid account of Fisher's approach. and ask how this may be described by means of a differential equations system

$$
\dot{X}=C \cdot(X-\bar{X}), X, \dot{X} \in \mathbb{R}^{m} ;
$$

$C$ of order $n \times n$ and rank $n, \bar{X}=B \cdot \bar{x}$;

in the sense that the true movement of $X$, which is $B(\dot{x})=$ $B\{A(x-\bar{x})\}$ is correctly described by the macro system (M).

The dynamic setting changes the aggregation problem because it adds restrictions: the aggregation matrix which associates $\dot{X}$ with $\dot{x}$ must be the same as that which associates $X$ with $x$, namely $B$ from (A). In contrast, Fisher's aggregation problem would allow for different aggregators linking the members of the pairs $(X, x)$ and $(Y, y)$. The main point is, however, that the true micro model describes the movements of the microeconomic state vector $x \in \mathbb{R}^{n}$ over time. If it tends to a subspace of $\mathbb{R}^{n}$ sufficiently fast, it will be sufficient to restrict the aggregation problem to this subspace rather than being compelled to assume a certain distribution of $x$ over $\mathbb{R}^{n}$, like in the Fisherian approach. The distribution of $x$ over $\mathbb{R}^{n}$ is, so to speak, endogenous here.

Further, if (S) stands for an economic model, it should be structurally stable, i. e. small changes of the model should not change its qualitative behavior. This implies the following assumption (Markus, 1961, Theorem 4):

Assumption: All eigenvalues of $A$ are distinct.

This assumption will turn out to be most convenient analytically.

The paper is organized as follows: Sect. 2 introduces the notion of the aggregation set, Sect. 3 discusses exact aggregation, Sect. 4 discusses approximate aggregation, starting either from an aggregation set (Sect. 4.1) or from selected eigenvalues (Sects. 4.2-4.4). The paper concludes with a discussion of the intuition underlying the present approach and the interpretation of macro relations in general. The appendix gives a numerical example which is intended to illustrate how the procedure works from a more technical viewpoint. 


\section{The Aggregation Set}

Consider first the case of a given microeconomic system (S), a given aggregation rule $(\mathrm{A})$ and a given macroeconomic system $(\mathrm{M})$. A first question to ask is in which sense the macroeconomic system (M) may describe the behavior of the underlying microeconomic system. This is the SAM Problem. ${ }^{5}$

Look first at the set of microeconomic states where the macro system describes the movement of the macro variables exactly. The true movement of the macro state is $\dot{X}=B \cdot \dot{x}$ and hence

$$
\dot{X}=B \cdot A \cdot(x-\bar{x}) \text {. }
$$

On the other hand, the macro model would give $\dot{X}=C \cdot(X-\bar{X})$. In conjunction with the aggregation rule this leads to

$$
\dot{X}=C \cdot B(x-\bar{x}) \text {. }
$$

The set of micro states $x$ where (1) and (2) give the same result is this set of states where the macro system describes the movement of the macro state exactly. Denote this the aggregation set $\Sigma:{ }^{6}$

$$
\Sigma=\left\{x \in \mathbb{R}^{n} \mid B \cdot A \cdot(x-\bar{x})=C \cdot B(x-\bar{x})\right\} .
$$

Two problems may be stated now. The first is: under which conditions will the aggregation set coincide with the entire state space $\mathbb{R}^{n}$ ? If these conditions are met, the macro system will describe the movement of the macro variables always exactly. Within the present context, we may term this the problem of exact aggregation. Sect. 3 will deal with this problem.

The second problem - the problem of approximate aggregation - arises if exact aggregation is impossible. In this case, we may ask how the macro model should be devised such that the micro state approaches the aggregation set as quickly as possible. This would minimize the aggregation error in the following sense. If the microeconomic state is close to the aggregation set, the macro model will offer a good description of the behavior of the macro variables. On the aggregation set, exact aggregation will be obtained. If the micro state approaches the aggregation set fast, aggregation will be approximately correct most of the time and the transitory phases where the micro states are far away from the

5 Cf. Schlicht (1977, p. $56 ; 1985$ a, p. 66)

6 Cf. Schlicht (1985a, p. 66). The notion of the aggregation set in Schlicht $(1977$, p. 57$)$ is slightly different. aggregation set and aggregation is poor are minimized. This issue is dealt with in Sect. 4 below.

\section{Exact Aggregation}

Exact aggregation is obtained if the aggregation set coincides with the state space $\mathbb{R}^{n}$. This implies the following equality which links the macro model with the aggregation rule and the micro model:

$$
B \cdot A=C \cdot B \text {. }
$$

This leads to the following proposition. ${ }^{7}$

Proposition 1: Exact local aggregation is possible if and only if the aggregation matrix $B$ is a nonsingular transformation of $m$ left-hand eigenvectors of the micro system matrix $A$. The macro model is then given by

$$
C=B A B^{\prime}\left(B B^{\prime}\right)^{-1} \text {. }
$$

Each eigenvalue of $C$ is also an eigenvalue of $A$.

Proof:

1. Assume first that $B$ is a nonsingular transformation $T$ of $m$ of the left-hand eigenvector of $A$ which make up the $m \times n$-matrix $L$. We show that this implies Eq. (4). Since

$$
B=T \cdot L
$$

we may choose $T$ without loss of generality such that $L^{\prime} L=I$ obtains. Denote the $m$ eigenvalues of $A$ which are associated with $L$ by $\Lambda_{m}=\operatorname{diag}\left(\lambda_{1}, \lambda_{2}, \ldots, \lambda_{m}\right)$. This leads to

$$
L \cdot A=\Lambda_{m} \cdot L \text {. }
$$

The matrix $B A$ can be evaluated now by means of (6) and (7) as

$$
B \cdot A=T \cdot \Lambda_{m} \cdot L .
$$

On the other hand, $C B$ can be evaluated from (5), (6), and (7) as

$$
C \cdot B=T \cdot \Lambda_{m} \cdot L \text {. }
$$

Eqs. (8) and (9) imply Eq. (4).

2. Assume next that (4) holds true. Since $A$ and $B$ are of full rank, we can solve for $C$ and obtain (5).

7 The idea to use eigenvectors as aggregators is due to Loch (1985). 
3. The last part of the proposition is covered by the more general Prop. 3 which will be stated and proved in Sect. 4 below. Q.E.D.

It is thus always possible to aggregate exactly by means of some of the left-hand eigenvectors of the micro system matrix $A$, but often this will not be particularly useful for the following reasons: first, we often want to determine the aggregation rule, say a certain index - without reference to and independently of the micro system, and secondly, aggregation by means of eigenvectors might be quite problematic if these turn out to comprise negative or complex components. If the aggregation matrix $B$ is fixed in advance without reference to the micro system $A$ and happens to be unattainable as a transformation of some left-hand eigenvectors of $A$, Prop. 1 tells us that exact aggregation cannot be obtained. This leads us to the problem of approximate aggregation.

\section{Approximate Aggregation}

\subsection{Selecting an Aggregation Set}

Consider the aggregation problem for an arbitrarily given aggregation matrix $B$ and a given micro system $A$. The following proposition provides a starting point for the consideration of this problem.

Proposition 2: Let $N$ be a real or complex $n \times m$-matrix of full rank. For the aggregate model defined by

$$
C=B A N(B N)^{-1}
$$

all $x$ with the representation

$$
x=\bar{x}+N \beta \text { for some } \beta \in \mathbb{C}^{m}
$$

are contained in the aggregation set. ${ }^{8}$

Proof: For any $x$ satisfying (11) we have

$$
\begin{gathered}
C B(x-\bar{x})=C B N \beta= \\
=B A N(B N)^{-1} B N \beta=B A N \beta=B A(x-\bar{x})
\end{gathered}
$$

Q. E. D.

$\mathbb{C}$ denotes the set of complex numbers.
This proposition establishes that we can define an appropriate macro model for any subspace of $\mathbb{R}^{m}$ given by (11). The aggregation set may be larger, however, than the set with representation (11), e. g. in the cases studied in Sect. 3 above when $B$ happens to be a transformation of some left-hand eigenvectors of $A$.

According to Prop. 2, the aggregation problem can be viewed as a problem of selecting an appropriate aggregation set.

\subsection{Selecting Eigenvalues}

The micro dynamics are characterized by the eigenvalues of $A$ and the macro dynamics are characterized by the eigenvalues of $C$. So the question arises of how the "micro eigenvalues" and the "macro eigenvalues" are interlinked. This question gives rise to the following proposition.

Proposition 3: All eigenvalues of $C$ are also eigenvalues of $A$.

Proof: Let $\lambda$ be an eigenvalue of $C$. This implies $\operatorname{det}(C-\lambda I)=0$ and hence $\operatorname{det}\left[B A N(B N)^{-1}-\lambda I\right]=0, \operatorname{det}(B A N-\lambda B N)=0$, det $B(A-\lambda I) N=0$. If $(A-\lambda I)$ were of full rank, $B(A-\lambda I) N$ would be of full rank, too, and the last equation could not be true. Hence $\lambda$ must be an eigenvalue of $A$.

Q. E. D.

The macro model reflects thus the dynamics of the micro model in so far as both models have common eigenvalues, but the macro model can take account only of $m$ out of the $n$ eigenvalues of the micro model. This gives rise to the question whether we can define a macro model by selecting some eigenvalues of $A$ rather than by selecting an aggregation set.

In a sense, the problem is easy: we may select some eigenvalues of $A$ and pick an arbitrary matrix $C$ with just these eigenvalues. The corresponding aggregation set can then be determined. The macro model $C$ determined in such an arbitrary fashion will, however, have only a loose connection with the underlying micro model, and the associated aggregation set may contain only the equilibrium.

We should approach the problem more carefully, therefore, and select the eigenvalues and the macro model such that it describes the macro dynamics as correctly as possible and leads to an appropriate aggregation set. We should start from more explicit dynamic considerations.

Denote the eigenvalues of $A$ by $\left(\lambda_{1}, \lambda_{2}, \ldots, \lambda_{n}\right)$ and select $m$ of them, say $\left(\lambda_{1}, \lambda_{2}, \ldots, \lambda_{m}\right)$, as eigenvalues of the macro model. 
Write

$$
\begin{gathered}
\Lambda=\operatorname{diag}\left(\lambda_{1}, \lambda_{2}, \ldots, \lambda_{n}\right), \\
\Lambda_{1}=\operatorname{diag}\left(\lambda_{1}, \lambda_{2}, \ldots, \lambda_{m}, 0,0, \ldots, 0\right), \\
\Lambda_{2}=\operatorname{diag}\left(0,0, \ldots, 0, \lambda_{m+1}, \lambda_{m+2}, \ldots, \lambda_{n}\right), \\
\Lambda=\Lambda_{1}+\Lambda_{2} .
\end{gathered}
$$

Consider now the micro model and its solutions

$$
x(t)=\bar{x}+\exp (A \cdot t) \cdot(x(0)-\bar{x}) .
$$

Since we have assumed all eigenvalues of $A$ to be distinct, $A$ has the representation

$$
A=T^{-1} \Lambda T
$$

with $T$ as the $n \times n$-matrix of the $n$ left-hand eigenvectors of $A$ and $T^{-1}$ as the $n \times n$-matrix of the $n$ right-hand eigenvectors of $A .^{9}$ Eqs. (13)-(15) imply

$$
x(t)=\bar{x}+T^{-1}\left[\exp \left(\Lambda_{1} \cdot t\right) \cdot \exp \left(\Lambda_{2} \cdot t\right)\right] T \cdot(x(0)-\bar{x}) .
$$

Since the macro model will neglect all solutions governed by $\Lambda_{2}$, $\Lambda$ is to be splitted into $\Lambda_{1}$ and $\Lambda_{2}$ in such a way that $\Lambda_{2}$ is not dominant in (16). A straightforward assumption is

$$
\text { re }\left(\lambda_{i}\right)<0, i=m+1, m+2, \ldots, n,
$$

i. e. that the movements governed by $\Lambda_{2}$ are damped. This implies

$$
\lim _{t \rightarrow \infty} e^{\Lambda_{2} t}=\left[\begin{array}{ll}
I & 0 \\
0 & 0
\end{array}\right]=: J_{1}
$$

Define now the idempotent matrix ${ }^{10}$

$$
D:=T^{-1} J_{1} T
$$

and associate with each $x(t)$ the approximation

$$
\tilde{x}(t):=\bar{x}+D(x(t)-\bar{x}) .
$$

Since $\tilde{x}(t)$ satisfies

$$
\dot{\tilde{x}}(t)=A(\tilde{x}(0)-\bar{x})
$$

it gives a class of solutions to the micro model evolving in the space spanned by $D$.

\footnotetext{
9 Cf. e. g. Bellmann (1960, p. 188)

10 Note that $D$ is unique since $(P Z T)^{-1} J_{1}(P Z T)=T \quad J_{1} T$ for any diagonal matrix $Z$ and any permutation matrix $P$.
}

Furthermore, $\tilde{x}(t)$ satisfies

$$
\dot{\tilde{x}}(t)=T^{-} \Lambda_{1} T(\tilde{x}(0)-\bar{x} .
$$

and has the general solution

$$
\tilde{x}(t)=T^{-1} e^{\Lambda_{1} t} T(\tilde{x}(0) \quad \bar{x})=T^{-} \quad T(x(0)-\bar{x})
$$

The error

$$
x(t)-\tilde{x}(t)=T^{-1} e^{\Lambda_{2} t} T(x(0)-\bar{x})
$$

tends to zero. Hence $\tilde{x}(t)$ can be viewed as an approximation to $x(t)$ involving only the first $m$ eigenvalues.

Since $D$ is of rank $m$, it can be decomposed as

$$
D=N E
$$

with $N$ of order $(n \times m), E$ of order $(m \times n)$ and $r(N)=r(E)=m$. By taking $\beta=E(x-\bar{x})$, all $\tilde{x}$ can be represented by

$$
\tilde{x}=\bar{x}+N \beta
$$

and Prop. 2 can be applied, leading to the macro model characterized by the matrix

$$
C=B A N(B N)^{-}
$$

The decomposition (25) is not unique, but this does not matter:

Proposition 4: All $N$ satisfying (25) lead to the same macro matrix $C$ as defined in (27).

Proof: Take any pair $N, E$ satisfying $D=N E$. Eq. (27) implies

and hence

$$
C B N E=B A N E
$$

$$
C B D B^{\prime}=B A D B^{\prime} \text {. }
$$

Since $B D B^{\prime}=\left(B T^{-1} I_{1}\right)\left(I_{1} T B^{\prime}\right)=(H, 0)\left(\begin{array}{l}K \\ O\end{array}\right)=H K$ with $H$ and $K$ of full rank, $B D B^{\prime}$ has full rank and $C$ satisfies

$$
C=B A D B \quad B D B
$$

and is, hence, independent of the choice of $N$.

The representation (30) can be used to characterize $C$ in a perhaps more transparent way. Write

$$
T=\left(\begin{array}{l}
L \\
Q
\end{array}\right), T^{-1}=(R, V), \Lambda_{m}=\operatorname{diag}\left(\lambda_{1}, \ldots, \lambda_{m}\right)
$$


with $L$ and $R$ as comprising the left-hand and right-hand eigenvectors of $A$ associated with the eigenvalues $\lambda_{1}, \lambda_{2}, \ldots, \lambda_{m}$, respectively.

Then

and we obtain

$$
D=R L, \quad A D=R \Lambda_{m} L
$$

Proposition 5: The macro matrix is determined by

$$
C=B R \Lambda_{m} L B^{\prime}\left(B R L B^{\prime}\right)^{-1}
$$

with $\Lambda_{m}$ comprising the eigenvalues selected for the macro model and $L$ and $R$ comprising the associated left-hand and right-hand eigenvectors of $A$, respectively.

The associated aggregation set is given by

$$
\Sigma=\left\{x \in \mathbb{R}^{n} \mid x=\bar{x}+R L(x-\bar{x})\right\} .
$$

Proof: Eqs. (33) and (34) are obtained by inserting Eqs. (32) into (30) and (20), respectively. Eq. (28) implies $C B R=B R \Lambda_{m}$. Hence $B R$ is the matrix of right-hand eigenvectors of $C$ and $\Lambda_{m}$ is the matrix of the eigenvalues of the macro model.

Q.E.D.

\subsection{The Aggregation Procedure}

The aggregation set given in Prop. 5 is asymptotically stable if the neglected roots $\lambda_{m+1}, \ldots, \lambda_{n}$ have negative real parts. Hence the macro model describes a set of particular solutions of the micro model exactly towards which all other solutions are tending, and in this sense we have obtained an approximate macro description of the micro flow.

The absolute size of the real parts of the neglected eigenvalues $\lambda_{m+1}, \lambda_{m+2}, \ldots, \lambda_{n}$ thus govern the speed of convergency towards the aggregation set (34). This suggests the following procedure for selecting eigenvalues for the macro model.

Renumber the eigenvalues of $A$ according to the size of their real parts in descending order:

$$
\operatorname{re}\left(\lambda_{1}\right)>\operatorname{re}\left(\lambda_{2}\right)>\ldots>\operatorname{re}\left(\lambda_{n}\right) .
$$

If the micro model is to be reduced to $m$ dimensions, keep the first $m$ eigenvalues for the macro model and neglect the rest. Check, however, whether re $\left(\lambda_{m+1}\right)$ is negative - otherwise the macro model would not make sense and $m$ is to be increased.

Write $\Lambda_{m}=\operatorname{diag}\left(\lambda_{1}, \lambda_{2}, \ldots, \lambda_{m}\right)$ and determine the matrices $L$ and $R$ from $L \cdot A=\Lambda_{m} \cdot A$ and $A \cdot R=R \cdot \Lambda_{m}$. The macro model is then computed from Eq. (33) and the associated aggregation set is determined by Eq. (34).

This procedure makes sure that the aggregation set is approached as fast as possible, and in this sense, the above procedure leads to an optimal macro approximation of the underlying micro model.

\subsection{Is the Macro Model Real?}

The aggregation procedure described above may lead, however, to a complex macro matrix $C$, and this is unwarranted. Take for instance the case $n>1, m=1$ and $\lambda_{1}$ complex. Then $C=\left(\lambda_{1}\right)$ is complex according to (33). A macro model operating in the complex domain seems however to make not very much sense both economically and in terms of reduction in dimensions of the real underlying model which is, after all, the purpose of aggregation.

We have thus to make sure that $C$ is real. This may happen even if $\Lambda_{m}$ is complex. Take, for instance, the case that all suppressed roots (i. e. $\Lambda_{2}$ ) are real and denote the associated eigenvectors by $Q$ and $V$ as in (31). Then

$$
R L=I-V Q \text {. }
$$

If $\Lambda_{2}$ is real, $V$ and $Q$ are real and (36) implies that $D=R L$ is real, and (30) implies that $C$ is real.

More generally, the following proposition holds true.

Proposition 6: The macro matrix $C$ is real if and only if $\Lambda_{m}$ has only real and/or conjugate complex components.

Proof:

1. If $C$ is real, all eigenvalues of $C$ are either real or conjugate complex. According to Prop. 5, $\Lambda_{m}$ is the matrix of eigenvalues of $C$ and has, hence, only real and conjugate complex components.

2. Assume that $\Lambda_{m}$ has only real or conjugate complex components. Arrange the eigenvalues such that the first $k$ eigenvalues are real and that the remaining pairs $\left(\lambda_{k+1}, \lambda_{k+2}\right)$, $\left(\lambda_{k+3}, \lambda_{k+4}\right), \ldots,\left(\lambda_{m-1}, \lambda_{m}\right)$ are conjugate complex. Split the matrices $L$ and $R$ as defined in (31) into their real and imaginary parts and write

$$
L=L^{*}+i L^{* *}, R=R^{*}+i R^{* *}
$$

with $L^{*}, L^{* *}, R^{*}, R^{* *}$ real. Since the eigenvectors belonging to 
conjugate complex eigenvalues are conjugate complex, too, we can generate the matrices $L^{*}, L^{* *}, R^{*}, R^{* *}$ as follows. Define the matrix $\hat{L}^{*}$ as comprising the rows nos. $1,2, \ldots, k$ and $k+1, k+3$, $\ldots, m-1$ of $L^{*}$, define the matrix $\hat{L}^{* *}$ as comprising the rows nos. $k+1, k+3, \ldots, m-1$ of $L^{* *}$. Then

$$
L=Z^{*} \hat{L}^{*}+i Z^{* *} \hat{L}^{* *} \text {. }
$$

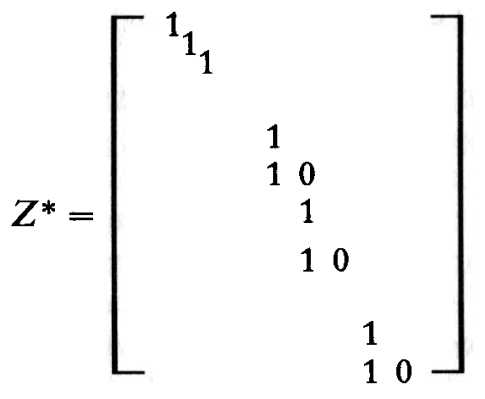

of order $m \times(m+k) / 2$ and

$$
Z^{* *}=\left[\begin{array}{rrrrr}
0 & & & & 0 \\
0 & & & & 0 \\
1 & & & & \\
-1 & 0 & & 0 & \\
& 1 & & & \\
& -1 & 0 & & \\
& & & 1 & \\
& & & -1 & 0
\end{array}\right]
$$

of order $m \times(m-k) / 2$.

Similarly, $R$ can be decomposed as

Hence

$$
R=\hat{R}^{*}\left(Z^{*}\right)^{\prime}+i \hat{R}^{* *}\left(Z^{* *}\right)^{\prime}
$$

$$
\begin{aligned}
R L= & \hat{R}^{*}\left(Z^{*}\right)^{\prime} Z^{*} \hat{L}^{*}+i \hat{R}\left(Z^{*}\right)^{\prime} Z^{* *} \hat{L}^{* *}+ \\
& +i \hat{R}^{* *}\left(Z^{* *}\right)^{\prime} Z^{*} \hat{L}^{*}-\hat{R}^{* *}\left(Z^{* *}\right)^{\prime} Z^{* *} \hat{L}^{* *}
\end{aligned}
$$

Since $\left(Z^{*}\right)^{\prime} Z^{* *}$ and $\left(Z^{* *}\right)^{\prime} Z^{*}$ are zero, $D$ is real. Eq. (30) implies that $C$ is real.

Q. E. D.

As a corollary of the above proof we obtain furthermore

Proposition 7: If the macro matrix $C$ is real, the aggregation set is given by

$$
\Sigma=\left\{x \in \mathbb{R}^{n} \mid x=\bar{x}+D \mu, \mu \in \mathbb{R}^{n}\right\}
$$

with $D$. The macro image of the aggregation set is the entire macro state space $\mathbb{R}^{m}$.

Proof: If $C$ is real, (41) implies that $D$ is real. Since $D$ is idempotent, all $x=\bar{x}+D \mu$ satisfy $x-\bar{x}=D(x-\bar{x})$. This proves (42). The macro image of $\Sigma$ is defined by the condition $X=\bar{X}+B D \mu$ in the neighborhood of $\bar{X}$. $B D$ is of full rank which proves the second part of the proposition.

Q. E. D.

Props. 6 and 7 make clear that the proposed aggregation procedure will lead to a real macro matrix $C$ under the weakest possible condition, namely that the selected eigenvalues are conjugate complex and that the domain of the macro model comprises the entire $\mathbb{R}^{m}$

\section{Conclusion}

The view of aggregation expounded here is the following. Although it is not possible in general to give a macro description of a micro model over the entire state space of the micro model, it is possible to aggregate exactly over a subspace. This set of micro states where aggregation is possible forms the aggregation set. The macro model can be chosen now in such a way that it gives rise to an aggregation set which is approached by the micro movements as fast as possible. This is done technically by selecting appropriate eigenvalues of the micro model and deriving a macro model based on these eigenvalues. As a result, the macro model so derived will describe trajectories of the aggregate quantities towards which the "true" trajectories of these quantities, as generated by the micro model, are tending.

This general view of aggregation is by no means confined to the linear case explored in this paper. It may be understood as a simple generalization of the well-known moving equilibrium method (Schlicht, 1985 a, pp. 32, 67f.). The present paper amplifies on the formal aspects of this view in the linear case. The spirit of the results will hopefully carry over to the nonlinear case (Mak, 1988).

We should, however, keep aware that the issue of aggregating a given micro theory into a macro version is actually not the main point in aggregation theory. The aggregation problem is most blatantly present when we have a macro theory but not the underlying micro theory, and this condition is met by almost the entire body of macroeconomics, if not microeconomics (Schlicht, 1985 a, pp. 12, 
93-100). Aggregation theory should, I think, expound the meaning of macroeconomic theorizing and give us a guide for devising reasonable macro theories.

The interpretation of macroeconomic relations underlying the present approach is not a new one. It is, in fact, quite orthodox. Macro theorists have always been aware that macro relations built on a certain micro structure of the economy. These relations may be affected by "structural effects". John Maynard Keynes was quite explicit about that. When introducing the notion of effective demand, he wrote for instance: "... we assume that a given volume of effective demand has a particular distribution of this demand between different products uniquely associated with it." 11 The understanding was that the economic system generates a certain structure - a particular distribution of demand between different products - which we may presuppose when building aggregative theories. The idea may be expressed in terms of the present analysis by saying that there are strong micro forces which push the micro states towards the aggregation set such that we can confine our attention to the states in the aggregation set. This permits aggregative analysis. The approach proposed here provides, in this sense, a defense of orthodox thinking.

\section{Appendix: An Example}

In order to give some flesh to the above considerations I consider the following example. I take a nonlinear model, namely a variant of Stiglitz' (1969) distribution model and consider a local linear approximation around equilibrium. For ease of computation, and also in order to illustrate how I would implement the method in practice, I consider the discrete time case, but I take a sufficiently small step width to assure that the qualitative behavior will not differ from the continuous time case discussed in the paper.

The population is divided into $n$ groups with savings ratios $s_{i}$, $i=1, \ldots, n$. Each group grows with rate $g$ and comprises a fraction $\alpha_{i}, i=1,2, \ldots, n$ of the population. Per capita wealth of group $i$ is denoted by $c_{i}$, and all individuals receive the same wage rate $w$.

11 Keynes (1936, p. 43). For more references to Keynes' view of aggregation, see Schlicht (1977, p. 104). Note, however, that Keynes' notion of effective demand is different from current usage. It refers to the intersection of aggregate supply and aggregate demand; see Schlicht (1979).
Denote by $r$ the rate of profit. Per capita wealth holdings increase by $s_{i}\left(w+r c_{i}\right)$ through savings and decrease by $g \cdot c_{i}$ through population growth. This leads to

$$
\dot{c}_{i}=s_{i}\left(w+r \cdot c_{i}\right)-g \cdot c_{i} .
$$

Capital intensity is ${ }^{12}$

$$
k=\sum_{i} \alpha_{i} c_{i}=\alpha c
$$

with

$$
\alpha=\left(\alpha_{1}, \ldots, \alpha_{n}\right), c=\left(c_{1}, \ldots, c_{n}\right) .
$$

Wages and profits are determined according to marginal productivity theory from a neoclassical production function

$y=f(k), f(0)=0, f^{\prime}(0)=\infty, f^{\prime}(\infty)=0, f^{\prime}>0, f^{\prime \prime}<0$

relating average per capita income $y$ to capital intensity $k$,

$$
r=f^{\prime}(k), w=f(k)-k \cdot f^{\prime}(k) .
$$

Hence (43) can be rewritten as

$$
\dot{c}_{i}=s_{i}\left(f(\alpha c)+f^{\prime}(\alpha c)\left(c_{i}-\alpha c\right)\right)-g \cdot c_{i} .
$$

This is a differential equations system with unique locally stable equilibrium $\bar{c}=\left(\bar{c}_{1}, \ldots, \bar{c}_{n}\right)$ and associated equilibrium capital intensity $\bar{k}=\alpha \bar{c}$ (see Schlicht, 1985 a, p. 89).

Our aim is to describe the movement of (47) around equilibrium by means of the Solow-type differential equation

$$
\dot{k}=s(k) f(k)-g \cdot k
$$

by using the aggregation rule (44).

The function $s(k)$ gives the aggregate savings ratio, and we have to determine this function around equilibrium along the lines described in the last section.

In order to obtain a correct equilibrium solution, we require

$$
s(\bar{k}) \cdot f(\bar{k})-g \cdot \bar{k}=0
$$

in equilibrium, i. e. for $\bar{k}=\alpha \bar{c}$. This implies the equilibrium savings ratio

$$
\bar{s}:=s(\bar{k})=g \cdot \bar{k} / f(\bar{k})
$$

${ }^{12}$ In the following I use the apostroph to indicate derivatives of a function rather than transposition of a matrix as has been the understanding in the main part of the paper. In this appendix, the vector product $\alpha \cdot c$ is always to be understood as an inner product. 
Linearizing (48) around equilibrium leads to

$$
\text { Hence } \quad \begin{aligned}
\dot{k}= & \left\{s^{\prime}(\bar{k}) \cdot f(\bar{k})+s(\bar{k}) \cdot f^{\prime}(\bar{k})-g\right\}(k-\bar{k}) . \\
\lambda & \lambda=s^{\prime}(\bar{k}) \cdot f(\bar{k})+g \cdot \bar{k} \cdot f^{\prime}(\bar{k}) / f(\bar{k})-g
\end{aligned}
$$

is the eigenvalue of the macro system which is to be equated to the smallest eigenvalue of the true system (47). This determines the macro savings function around equilibrium as

with

$$
s(k)=s(\bar{k})+s^{\prime}(\bar{k})(k-\bar{k})
$$

$$
s^{\prime}(\bar{k})=\frac{1}{f(k)}\left(\lambda+g\left(1-\bar{k} f^{\prime}(\bar{k}) / f(\bar{k})\right)\right) .
$$

Note that the macro savings function is dependent upon $k, g$ and the shape of the production function whereas the individual savings ratios are constant. ${ }^{13}$

As a numerical example take $f$ as CES with $\sigma=0.5, \alpha=0.6$, $\gamma=1$. Take $g=0.07, n=5, s=(0.05,0.1,0.2,0.25,0.3), \alpha=(0.1$, $0.3,0.35,0.2,0.05)$. The resulting equilibrium of $(39)$ has $\bar{k}=4.8922, f(\bar{k})=1.9134, f^{\prime}(\bar{k})=0.0918$ and the minimal eigenvalue -0.0654 . Hence we obtain

$$
s(k)=0.1790-0.0086(k-\bar{k})
$$

as the macro savings function. Inserting this into (48) and rewriting it as a difference equation gives the macro model

$$
k_{t+1}=\frac{1}{1+g}\left(k_{t}+s\left(k_{t}\right) \cdot f\left(k_{t}\right)\right) \text {. }
$$

The behavior of this model can be compared with the behavior of the discrete-time version of (47) which is

$$
c_{i, t+1}=\frac{1}{1+g}\left(c_{i, t}+s_{i}\left(f\left(\alpha c_{t}\right)+f^{\prime}\left(\alpha c_{t}\right)\left(c_{i t}-\alpha c_{t}\right)\right) .\right.
$$

This is done as follows: each $\bar{c}_{i}$ is disturbed by a uniformely distribution random disturbance $v_{i} \in(-0.5,+0.5)$. The resulting $c$ is normalized to $\alpha c=\bar{k}+1$ and taken as the initial value for (57). The time path of the corresponding $k_{t}=\alpha c_{t}$ is computed for alternative starting values generated as described above. Furthermore the solution of the macro model (56) is computed with $k_{0}=\bar{k}+1$. Differences between the various micro solutions and the macro

13 This phenomenon has been termed "context dependency of macro relations" in Schlicht (1976, p. 75 f.; 1985 a, p. 78 f.).
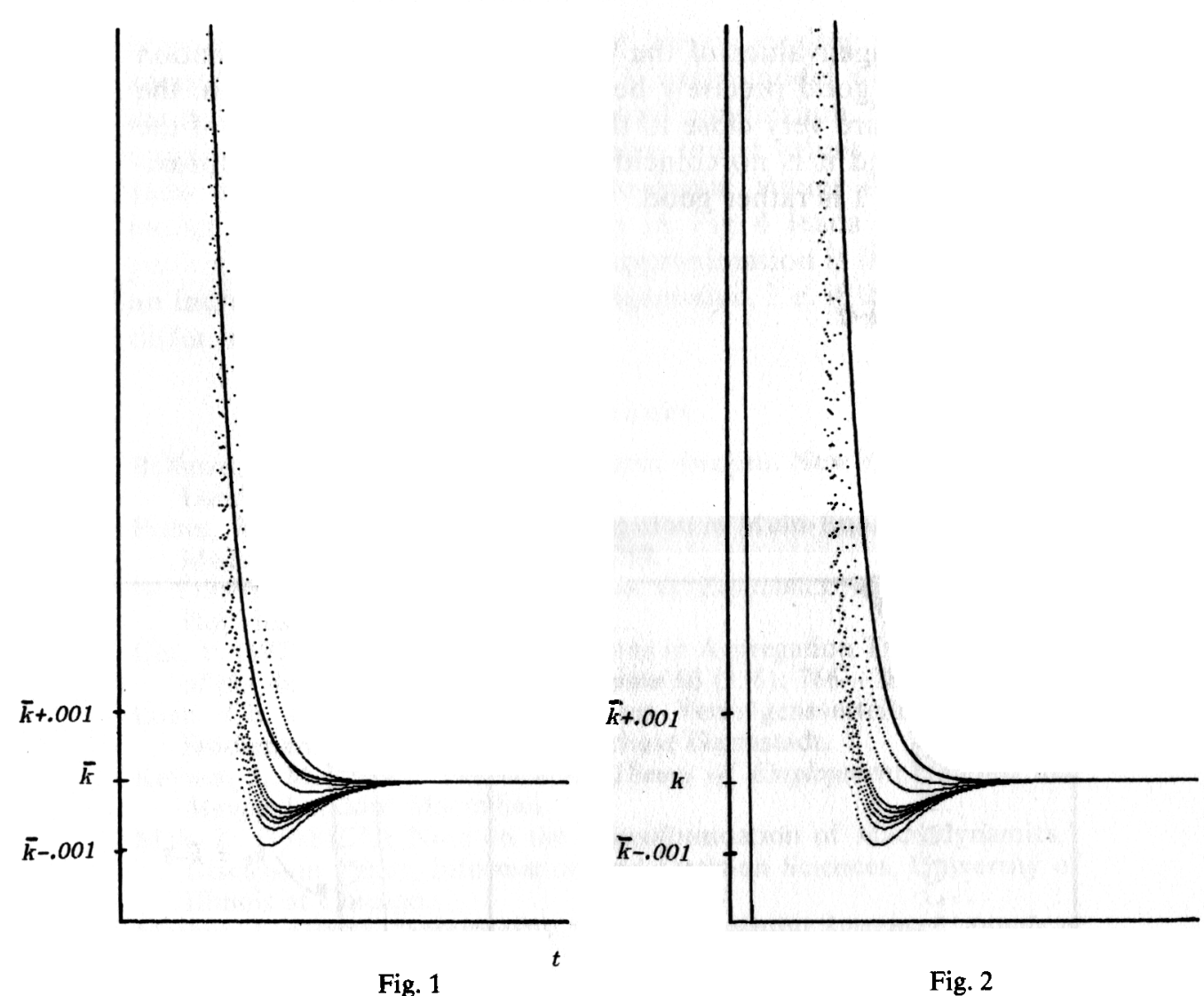

Fig. 2

solution turn out to be rather small but can be depicted under the "microscopic" view presented in Fig. 1, where the dotted curves give exact solutions for alternative initial values $c_{0}$ with $\alpha c_{0}=\bar{k}+1$ generated as described above, and the unbroken curve depicts the solution of the macro equation starting with $k_{0}=\bar{k}+1$. In Fig. 2 , the macro curve is shifted to the right. It can be seen that it coincides with the slowest true trajectory. This is not surprising, since the construction of the macro model is based on the smallest eigenvalue.

Since the eigenvalues of $A$ are very close in size, ranging in the interval $0.0654<|\lambda|<0.0704$, the approach to the slowest trajectory is not pronounced. But this should not lead to the conclusion that the proposed aggregation procedure makes sense only if the eigenvalues neglected in the macro model are considerably larger in size than the eigenvalues of the macro model: if the neglected roots are 
close to the eigenvalues of the macro model, the approximation will be rather good precisely because the speeds depicted in the macro model are very close to the true speeds of adaption of the true model, and it is no coincidence, therefore, that the approximation in Fig. 1 is rather good.

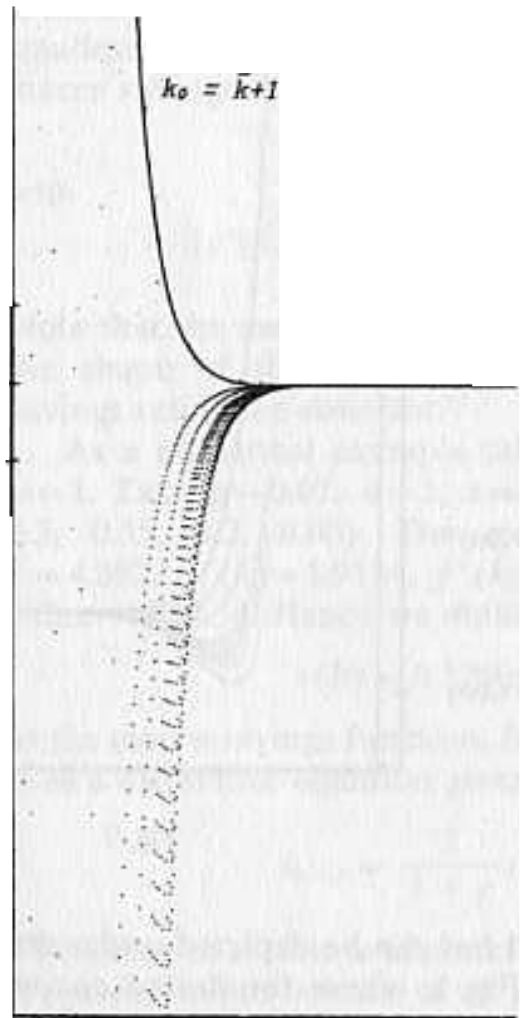

Fig. 3
Fig. 4

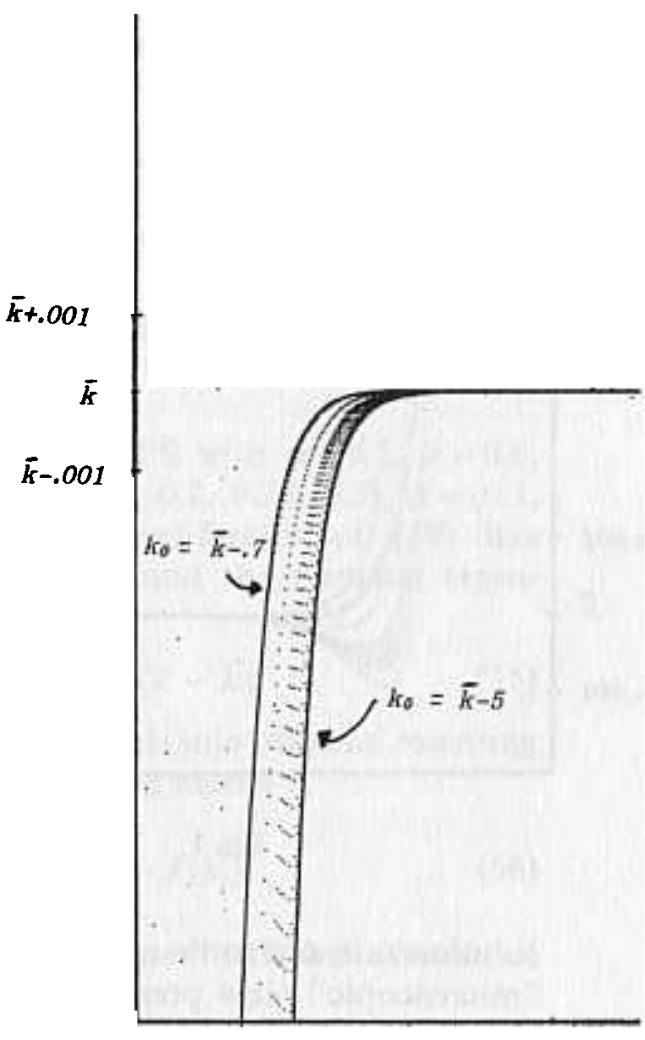

In order to illustrate this point, take $s=(0,0,0.0,1)$ and leave everything else unchanged. The eigenvalues of the Jacobian are now -0.1484 and -0.0654 , and Fig. 3 depicts the result. ${ }^{14}$ Fig. 3 seems

${ }^{14}$ The scale of Fig. 3 is the same as that of Figs. 1 and 2, but the origin is shifted downward. Furthermore, the smaller eigenvalue is of multiplicity 4 now, in deviation of what has been postulated in the paper, but the choice of savings ratios produces a maximal difference in eigenvalues, and this is the point which is to be illustrated here. not very convincing, but note that we are dealing with the local aggregation problem: we look for a macro model which describes the micro flow approximately around equilibrium, but we do not require that the micro and the macro initial values correspond to each other. Choosing appropriate initial values for the macro model $\left(k_{0}=\bar{k}-5\right.$ and $\left.k_{0}=\bar{k}-0.7\right)$ in Fig. 4 leads to an almost perfect fit. The reason is that the approximation is improving with an increasing dominance of one eigenvalue, i. e. if the eigenvalues differ in size.

\section{References}

Bellman, R. (1960): Introduction to Matrix Analysis. New York - Toronto London: McGraw-Hill.

Fisher, W. D. (1962): "Optimal Aggregation in Multi-Equation Prediction Models." Econometrica 30: 744-769.

- (1969): Clustering and Aggregation in Economics. Baltimore: Johns Hopkins Press.

Ijiri, Y (1971): "Fundamental Questions in Aggregation Theory." Journal of the American Statistical Association 66 (336): 766-782.

Loch, Ch. (1985): "Aggregation eines Vermögensverteilungsmodells." Studienarbeit, Technische Hochschule Darmstadt.

Keynes, J. M. (1936): The General Theory of Employment, Interest and Money. London: Macmillan.

Mak, K. (1988): "A Note on the Microfoundation of Macrodynamics." Discussion Paper, Information and Decision Sciences, University of Illinois at Chicago.

Markus, L. (1961): "Structurally Stable Differential Systems." Annals of Mathematics $73: 1-19$.

May, K. (1947): "Technological Change and Aggregation." Econometrica 15: $51-63$.

Schlicht, E. (1977): Grundlagen der ökonomischen Analyse. Reinbek bei Hamburg: Rowohlt.

- (1979): "On Keynesian Supply." Discussion Paper No. 54, Fakultät für Wirtschaftswissenschaften, Universität Bielefeld.

- (1985 a): Isolation and Aggregation in Economics. Berlin - Heidelberg New York - Tokyo: Springer-Verlag.

- (1985b): "The Local Aggregation Problem." Discussion Paper, Technische Hochschule Darmstadt.

Schneeweiß, H. (1965): "Das Aggregationsproblem." Statistische Hefte (Statistical Papers) 6: 1-26.

Sondermann, D. (1973): “Optimale Aggregation von großen Gleichungssystemen." Zeitschrift für Nationalökonomie 33: 235-250.

Stiglitz, J. E. (1969): "Distribution of Income and Wealth Among Individuals." Econometrica 37: 382-397.

Address of author: Prof. Dr. Ekkehart Schlicht, Department of Law and Economics, Technical University of Darmstadt, Residenzschloß, D-6100 Darmstadt, F.R.G. 